\title{
The BREATHE study: Breathing REtraining for Asthma - Trial of Home Exercises. A protocol summary of a randomised controlled trial
}

\author{
Anne Bruton', Sarah Kirby², Emily Arden-Close², Lynda Taylor³, Frances Webley3, \\ Steve George ${ }^{4}$, Lucy Yardley ${ }^{2}$, David Price ${ }^{5}$, Michael Moore ${ }^{6}$, Paul Little ${ }^{6}$, Stephen Holgate \\ Ratko Djukanovic ${ }^{7}$, Amanda J Lee ${ }^{5}$, James Raftery ${ }^{8}$, Maria Chorozoglou ${ }^{8}$, Jenny Versnel ${ }^{9}$, \\ Ian Pavord ${ }^{10}$, Mark Stafford-Watson ${ }^{11},{ }^{*}$ Mike Thomas $^{6}$

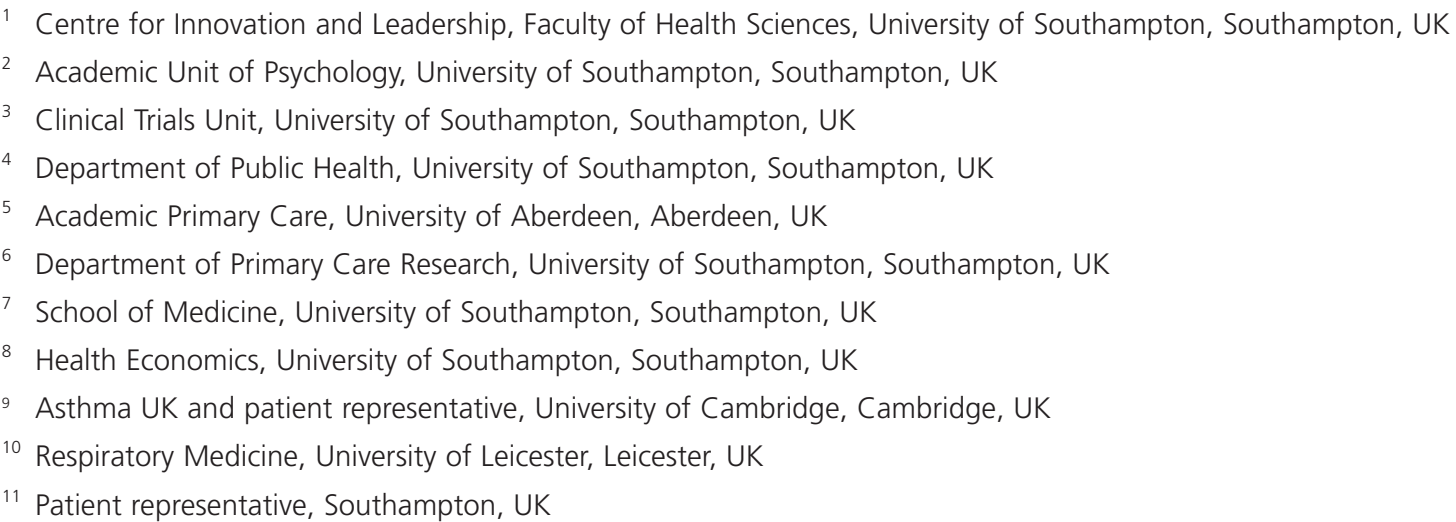

Received 5th March 2013; accepted 19th March 2013; online 30th May 2013

(C) 2013 Primary Care Respiratory Society UK. All rights reserved.

A Bruton et al. Prim Care Respir J 2013; 22(2): PS1-PS7

http://dx.doi.org/10.4104/pcrj.2013.00047

Keywords asthma, management, primary care, breathing retraining, home exercises, RCT

\section{Background}

Asthma affects five million people in the UK and costs the National Health Service (NHS) in excess of $f 1$ billion. Although pharmacotherapy is effective, outcomes remain suboptimal. Many patients have concerns about taking regular medication. Surveys of complementary and alternative medicine show high levels of use, with breathing modification techniques being among the most commonly used non-drug treatment. A systematic review found that $30 \%$ of patients reported using breathing techniques to control their symptoms. ${ }^{1}$

Asthma encompasses a variety of phenotypes, and different therapeutic approaches may be effective in different patients. ${ }^{2}$ Symptoms attributed to dysfunctional breathing are more frequent in people with asthma than in the general population., ${ }^{3,4}$ Recent studies have suggested clinically important effects for people with asthma from physiotherapist-administered breathing exercises. ${ }^{5-7}$
The most recent systematic review of the effectiveness of physiotherapist-taught breathing training for asthma, published in 2009, ${ }^{8}$ stated: 'Breathing exercises, incorporating reducing respiratory rate and/or tidal volume and relaxation training, should be offered to patients to help control the symptoms of asthma and improve quality of life (Grade A)'. Most of the studies contributing to the current evidence base have involved face-to-face interventions, but two preliminary studies have investigated the use of videotaped or DVD-provided instructional interventions, ${ }^{9,10}$ with some evidence that this modality may also be effective. If similar benefits can be achieved without face-to-face professional contact, the practical and economic implications of providing breathing training would be eased. This treatment could realistically be made available to many people with asthma who currently find it difficult to access it on the NHS.

In this study we will transfer the key components of the

\footnotetext{
* Corresponding author: Professor Mike Thomas, Professor of Primary Care Research, Department of Primary Care Research, University of Southampton, Aldermoor Health Centre, Aldermoor Close, Southampton, SO16 5ST, UK.

Tel: +44 (0)2380241050 Fax: +44 (0)2380701125 E-mail: mikethomas@doctors.org.uk
} 
physiotherapist-delivered programme into a DVD format and compare the effects of this intervention with that of face-to-face physiotherapist training and with 'usual care'.

\section{Hypothesis}

Breathing training exercises taught by an educational package consisting of an instructional DVD and supporting booklet will result in clinically important improvements in asthma-related quality of life and in asthma control above 'usual care' and of a similar magnitude (but at a lower cost) to those resulting from face-to-face physiotherapist instruction.

\section{Objectives}

1. To produce a breathing training programme for people with asthma (aged 16-70 years), incorporating breathing exercise instruction in an audiovisual DVD format with supporting booklet.

2. To perform a randomised controlled trial on people with asthma over a 12-month period to assess the effect of the programme in comparison with 'usual care' and with that of face-to-face physiotherapist-led training of similar content on asthma-related health status, parameters of symptomatic and physiological asthma control, and asthma-related health resource use.

3. To perform a quantitative process evaluation on patientreported expectations of treatment, treatment experience, and adherence.

4. To perform a qualitative process evaluation on 12-15 participants in each arm of the trial, purposively sampled for diversity.

5. To estimate the cost-effectiveness of the breathing retraining interventions delivered by DVD and by face-to-face instruction using data collected from the trial and from the GP-held clinical records.

\section{Methods}

Phase 1: Development phase: transfer of programme to audiovisual media and qualitative piloting of materials

Patient educational materials will be developed by members of the team including physicians, physiotherapists, health psychologists, communications technology specialists, and patient representatives. A DVD and accompanying booklet will be created, consisting of: (1) a detailed explanation and illustration of how to carry out the exercises; and (2) motivational components, explaining the rationale for the exercises and addressing common doubts and concerns.

The materials will be piloted with a panel of 20-30 members of the target population, purposively sampled for diversity in terms of age, gender, education, and symptom profile. In audiorecorded face-to-face and telephone interviews, we will use open-ended questions to explore attitudes to the proposed treatment method in the context of health beliefs and then use 'think aloud' methods" to elicit spontaneous reactions to all proposed materials. We will modify the scripts as necessary, based on this feedback. After professional production of the DVD and booklet, we will send them out to members of our panel to view and then provide final feedback by face-to-face and telephone interviews.

\section{Phase 2: Randomised controlled clinical trial (RCT)}

Trial description

We will perform a pragmatic observer-blinded three-arm parallel group randomised controlled trial comparing the DVD intervention with face-to-face physiotherapy and a control arm receiving 'usual care' for adults with asthma (Box 1). The study is designed and powered to show that the DVD intervention is superior to 'usual care' and that the DVD intervention is equivalent (i.e. of comparable efficacy) to the face-to-face intervention that has already been shown to be effective. Three arms are needed to determine superiority of the DVD over 'usual care' and comparable efficacy to face-to-face physiotherapy using a 2:2:1 randomisation plan. As the focus of the study is on demonstrating superiority of the new intervention (DVD) over 'usual care' and equivalence with an intervention already shown to be effective (face-to-face physiotherapy), the statistical power, logistics and expense of the study are improved by having a lower proportion of volunteers in the already tested arm.

Eligible patients will be sent a study invitation letter and asked to complete the mini-Asthma Quality of Life Questionnaire (AQLQ). ${ }^{12}$ Only those with impaired health status (AQLQ score $<5.5$ ) will be recruited. In previous work and other studies, $75 \%$ or more or people with asthma treated in the community have impaired health status.

Baseline assessment will consist of recording clinical data (smoking status, asthma history, co-morbidities, medication, and exacerbation frequency), completion of questionnaires (diseasespecific health status (AQLQ), Nijmegen hyperventilation questionnaire, Asthma Control Questionnaire, generic health status (EuroQOL, EQ-5D); anxiety and depression scores (HAD questionnaire), and physiological measures (spirometry: forced expiratory volume in one second $\left(F E V_{1}\right)$, ratio of $F E V_{1}$ to forced vital capacity, peak expiratory flow rate) measured with standardised calibrated portable spirometer; fraction of exhaled nitric oxide measured with Aerocrine Mino portable monitor). Randomisation will occur through the University of Southampton Clinical Trials Unit telephone randomisation service by the study nurse telephoning the unit and arranging follow-up appointments.

All participants will be posted questionnaires at 3 and 6 months, and a final assessment visit will be scheduled for 12 months after randomisation. Those randomised to 'usual care' will receive no other additional attention. Those randomised to the DVD will be provided with the booklet and DVD; for those who do not have a DVD player at home, one will be provided. Those randomised to the face-to-face physiotherapy arm will receive three short sessions of contact with a respiratory physiotherapist for breathing training. These sessions will be approximately 30-40 mins long and will be held at the GP surgery, the patient's home, or the local hospital depending on convenience and preference, and will occur at roughly 2-weekly intervals following randomisation.

The final 12-month assessment will be performed by a different study nurse blinded to the subject's randomisation group. The assessment will consist of the same clinical, questionnaire, and physiological measurements performed at baseline, plus a short 
Box 1. Randomised controlled trial

\section{Trial design}

Pragmatic observer blinded three-arm parallel-group randomised controlled trial comparing breathing retraining through a DVD with face-to-face physiotherapy and a 'control' of usual care for adults with asthma and impaired health status.

\section{Sample size}

Total of 585 participants, 234 in the DVD arm, 234 in the usual care arm, and 117 in the face-to-face physiotherapy arm. Inclusion criteria

- Full practice registration for a minimum of 12 months prior to enrollment

- Age 16-70 years

- Physician-diagnosed asthma in medical record

- $\quad \geq 1$ anti-asthma medication prescriptions in the previous year

- Impaired asthma-related health status (Asthma Quality of Life Questionnaire (AQLQ) score <5.5)

- Informed consent

\section{Exclusion criteria}

- Asthma judged at the baseline assessment to be dangerously unstable and in need of urgent medical review

- Chronic obstructive pulmonary disease (COPD) with forced expiratory volume in one second (FEV 1 ) $<60 \%$ predicted, to exclude those with overlapping asthma and COPD with more severe fixed airflow obstruction

We aim to allow broad entry criteria (with inclusion of smokers and not insisting on physiological demonstration of reversible airflow obstruction) in order to allow generalisability of research findings to mild-to-moderate UK asthma populations treated in primary care NHS practice.

\section{Duration of intervention}

- Face-to-face physiotherapy arm: three sessions lasting 30-40 mins at approximately two-weekly intervals

- DVD arm: for use by participants at own home as convenient

\section{Primary trial endpoint}

- Analysis of the between-group (intention-to-treat (ITT)) change in asthma-specific health status (AQLQ (short version) score) Secondary trial endpoints

- Asthma Control Questionnaire score; lung function (FEV1, ratio of FEV1 to forced vital capacity, peak expiratory flow rate)

- Fraction of exhaled nitric oxide

- Health status (EuroQOL)

- Anxiety and depression scores (HAD questionnaire; Hyperventilation (Nijmegen) questionnaire

- Oral corticosteroid courses

- Bronchodilator use

- Asthma-related health resource use

- Smoking status

- Cost effectiveness/utility

- Patient-reported process evaluations (questionnaires)

- Estimates of adherence (use of exercises)

Total number of sites: 20-30 GP practices

questionnaire exploring subject perception and experiences of being in the trial and adherence. All statistical analyses will be performed blinded to randomisation group.

Sample size justification

For the equivalence study (DVD vs. face-to-face physiotherapy), using Nquery 7.0: Sample sizes of 210 in the DVD breathing retraining group and 105 in the face-to-face physiotherapy group are required to assess treatment equivalence with $90 \%$ power using an equivalence boundary for AQLQ of 0.3. This assumes that the expected between-group difference in mean AQLQ is 0 , a two-tailed $5 \%$ significance level, common standard deviation for AQLQ of 0.77 and a lower/upper limit of $-0.3 /+0.3$ for the $95 \%$ confidence interval of the between-group difference in AQLQ.

For the superiority study (DVD vs. 'usual care'), a two-group t-test with a $5 \%$ one-sided significance level will have $92 \%$ power to detect a difference in mean AQLQ of 0.38 or greater, assuming that the common standard deviation is 1.03, when the sample sizes are 210 in the DVD breathing retraining and 210 in the 'usual care' groups.

\section{Qualitative process evaluation}

We will perform an open-ended qualitative study to evaluate patient experiences of the trial and identify factors that may have influenced 
trial outcomes. We will carry out telephone interviews with 12-15 patients in each arm of the trial, purposively sampled for diversity and seeking to ensure representation of participants with poor adherence or outcomes. The interviews will be carried after the 3month follow-up questionnaire evaluation. All data will be tape recorded, transcribed, and analysed inductively by thematic analysis. Quantitative process evaluation

Immediately after randomisation, participants randomised to the treatment groups (DVD and face-to-face physiotherapy) will be shown the booklet and asked to complete a questionnaire assessing their beliefs about asthma and their first impressions of the treatment. At the 3-month follow-up, participants in the treatment groups will be asked to complete brief questionnaires relating to their experiences of treatment, adherence to treatment, and perceived barriers to adherence. Questions to assess whether participants continued with the treatment will be included at the 6and 12-month follow-ups.

The schedule of observations and procedures is shown in Appendix 1 and the trial flow diagram is shown in Appendix 2.

\section{Phase 3: Health economic evaluation}

The resources needed to design and develop the DVD intervention will be recorded. Information will be collected on all asthma-related healthcare costs for the 1-year follow-up period using medical records. Respondent questionnaires will be completed to quantify time off work/school and expenses. NHS and societal costs will be estimated, allowing estimates of the incremental costs of the breathing DVD and face-to-face physiotherapy programmes compared with 'usual care'. Health-related quality of life will be assessed using the EuroQol (EQ5D) $)^{13}$ and used to generate qualityadjusted life years.

\section{Handling editor Anthony D'Urzo}

Conflicts of interest The authors declare that they have no conflicts of interest in relation to this protocol. MT is an Associate Editor of the PCRJ, but was not involved in the editorial review of, nor the decision to publish, this article.

Contributorship MT drafted the paper with contribution and approval from all authors. All authors have been and are actively involved in the study in different capacities; $A B$ is the physiotherapy lead, SK and EA-C are involved in the development of materials and the qualitative evaluations, LT and SW manage the trial; SG, LY, DP, MM, PL, SH, RD and IP have helped shape the study and are trial management group members, AL is the lead statistician, JR and MC lead the health economic evaluation, JV represents Asthma UK on the TMG and provides PPI input, MS-W is the PPI lead.

Funding This trial is funded by the NIHR Health Technology Assessment programme (HTA project number 09/104/19).

Sponsor University of Southampton

Protocol registration International Standard Randomised Controlled Trials Number Registration database: ISRCTN 88318003. UK Clinical Research Network database: 12934.

\section{References}

1. Ernst E. Breathing techniques: adjunctive treatment modalities for asthma? A systematic review. Eur Respir J 2000;15(5):969-72. http://dx.doi.org/10.1183/09031936.00.15596900

2. Anderson GP. Endotyping asthma: new insights into key pathogenic mechanisms in a complex, heterogeneous disease. Lancet 2008;372(9643):1107-19. http://dx.doi.org/10.1016/S0140-6736(08)61452-X

3. Thomas M, McKinley RK, Freeman E, Foy C. Prevalence of dysfunctional breathing in patients treated for asthma in primary care: cross sectional survey. BMJ 2001;322(7294):1098-100. http://dx.doi.org/10.1136/bmj.322.7294.1098

4. Thomas M, McKinley RK, Freeman E, Foy C, Price D. The prevalence of dysfunctional breathing in adults in the community with and without asthma. Prim Care Respir J 2005;14(2):78-82. http://dx.doi.org/10.1016/j.pcrj.2004.10.007

5. Thomas M, McKinley RK, Mellor S, et al. Breathing exercises for asthma: a randomised controlled trial. Thorax 2009;64(1):55-61. http://dx.doi.org/10.1136/thx.2008.100867

6. Holloway EA, West RJ. Integrated breathing and relaxation training (the Papworth method) for adults with asthma in primary care: a randomised controlled trial. Thorax 2007;62(12):1039-42. http://dx.doi.org/10.1136/thx.2006.076430

7. Thomas M, McKinley RK, Freeman E, Foy C, Prodger P, Price D. Breathing retraining for dysfunctional breathing in asthma: a randomised controlled trial. Thorax 2003;58(2):110-15. http://dx.doi.org/10.1136/thorax.58.2.110

8. Bott J, Blumenthal S, Buxton $\mathrm{M}$, et al. Guidelines for the physiotherapy management of the adult, medical, spontaneously breathing patient. Thorax 2009; 64(Suppl 1):i1-51. http://dx.doi.org/10.1136/thx.2008.110726

9. Opat AJ, Cohen MM, Bailey MJ, Abramson MJ. A clinical trial of the Buteyko breathing technique in asthma as taught by a video. J Asthma 2000;37(7):557-64 http://dx.doi.org/10.3109/02770900009090810.

10. Cooper S, Oborne J, Newton S, et al. Effect of two breathing exercises (Buteyko and pranayama) in asthma: a randomised controlled trial. Thorax 2003;58(8):6749. http://dx.doi.org/10.1136/thorax.58.8.674

11. van den Haak MJ, de Jong MDT, Schellens PJ. Evaluation of an informational web site: three variants of the think-aloud method compared. Technical Communication 2007; 54:58-71.

12. Juniper EF, Guyatt GH, Cox FM, Ferrie PJ, King DR. Development and validation of the Mini Asthma Quality of Life Questionnaire. Eur Respir J 1999;14(1):32-8. http://dx.doi.org/10.1034/j.1399-3003.1999.14a08.x

13. EuroQol Group. EuroQol: a new facility for the measurement of health-related quality of life. Health Policy 1990;16:199-20.

http://dx.doi.org/10.1016/0168-8510(90)90421-9

Available online at http://www.thepcrj.org 


\begin{tabular}{|c|c|c|c|c|c|c|c|}
\hline Visit: & $\begin{array}{c}\text { Pre } \\
\text { screen }\end{array}$ & $\begin{array}{c}\text { Baseline } \\
\text { assessment }\end{array}$ & $\begin{array}{l}3 \text { physio } \\
\text { sessions, } \\
2 \text { weekly } \\
\text { intervals }\end{array}$ & $\begin{array}{c}6-8 \\
\text { weeks } \\
\text { post } \\
\text { baseline }\end{array}$ & $\begin{array}{c}3 \text { month } \\
\text { Follow up } \\
\text { postal } \\
\text { questionnaires }\end{array}$ & $\begin{array}{c}6 \text { month } \\
\text { Follow up postal } \\
\text { questionnaires }\end{array}$ & $\begin{array}{c}12 \text { month } \\
\text { Follow up } \\
\text { visit } \\
\text { with } \\
\text { study } \\
\text { nurse } \\
\end{array}$ \\
\hline $\begin{array}{l}\text { Patient } \\
\text { Information } \\
\text { Sheet posted } \\
\end{array}$ & $x$ & & & & & & \\
\hline $\begin{array}{l}\text { mini-AQLQ } \\
\text { questionnaire } \\
\text { posted }\end{array}$ & $\mathrm{x}$ & & & & & & \\
\hline $\begin{array}{l}\text { Informed } \\
\text { Consent }\end{array}$ & & $\mathrm{x}$ & & & & & \\
\hline $\begin{array}{l}\text { Incl /Exclusion } \\
\text { Criteria }\end{array}$ & & $\mathrm{x}$ & & & & & \\
\hline $\begin{array}{l}\text { Questionnaires: } \\
\text { Mini-AQLQ, } \\
\text { Nijmegen } \\
\text { Hyperventilatio } \\
\text { n, HAD, ACQ, } \\
\text { EQ5D }\end{array}$ & & $x$ & & & & & $x$ \\
\hline $\begin{array}{l}\text { Expectancy : } \\
\text { Beliefs about } \\
\text { asthma, First } \\
\text { impressions } \\
\text { (Physio and DVD } \\
\text { only) }\end{array}$ & & $\begin{array}{c}\text { X } \\
\text { (after } \\
\text { randomisation) }\end{array}$ & & & & & \\
\hline $\begin{array}{l}\text { Questionnaires: } \\
\text { Mini AQLQ, HAD, } \\
\text { ACQ, EQ5D }\end{array}$ & & & & & $\mathrm{x}$ & $\mathrm{x}$ & \\
\hline $\begin{array}{l}\text { Questionnaires: } \\
\text { Treatment } \\
\text { experience } \\
\text { (physio only), } \\
\text { Treatment } \\
\text { experience (DVD } \\
\text { only), } \\
\text { Treatment } \\
\text { adherence } 3 \\
\text { month (Physio } \\
\text { and DVD only) }\end{array}$ & & & & & $\mathrm{x}$ & & \\
\hline $\begin{array}{l}\text { Treatment } \\
\text { adherence } 6 \& \\
12 \text { month } \\
\text { (Physio and DVD } \\
\text { only) }\end{array}$ & & & & & & $\mathrm{x}$ & $\mathrm{x}$ \\
\hline $\begin{array}{l}\text { Questionnaire: } \\
\text { Respondent } \\
\text { Costs } \\
\end{array}$ & & & & & $x$ & & $\mathrm{x}$ \\
\hline Clinical Details & & $x$ & & & & & $\mathrm{x}$ \\
\hline
\end{tabular}


Appendix 1: Schedule of observations and procedures continued

\begin{tabular}{|c|c|c|c|c|c|c|c|}
\hline Visit: & $\begin{array}{c}\text { Pre } \\
\text { screen }\end{array}$ & $\begin{array}{c}\text { Baseline } \\
\text { assessment }\end{array}$ & $\begin{array}{l}3 \text { physio } \\
\text { sessions, } \\
2 \text { weekly } \\
\text { intervals }\end{array}$ & $\begin{array}{c}\text { 6-8 } \\
\text { weeks } \\
\text { post } \\
\text { baseline }\end{array}$ & $\begin{array}{c}3 \text { month Follow } \\
\text { up } \\
\text { postal } \\
\text { questionnaires }\end{array}$ & $\begin{array}{l}6 \text { month Follow } \\
\text { up postal } \\
\text { questionnaires }\end{array}$ & $\begin{array}{l}12 \text { month } \\
\text { Follow up } \\
\text { visit } \\
\text { With study } \\
\text { nurse }\end{array}$ \\
\hline $\begin{array}{l}\text { Physiological } \\
\text { measurements: } \\
\text { Spirometry and } \\
\text { FENO }\end{array}$ & & $\mathrm{X}$ & & & & & $\mathrm{X}$ \\
\hline Randomisation & & $\mathrm{X}$ & & & & & \\
\hline $\begin{array}{l}\text { Provision of DVD } \\
\& \text { 'Booklet' Group } \\
1\end{array}$ & & $\mathrm{X}$ & & & & & \\
\hline $\begin{array}{l}\text { Face-to-face } \\
\text { Physiotherapy }\end{array}$ & & & $X$ & & & & \\
\hline $\begin{array}{l}\text { Process } \\
\text { Evaluation } \\
\text { Telephone } \\
\text { interview for } 12 \\
\text { to } 15 \text { patients in } \\
\text { each group }\end{array}$ & & & & $\mathrm{X}$ & & & \\
\hline $\begin{array}{l}\text { Medical Notes } \\
\text { Review }\end{array}$ & & & & & & & $\mathrm{X}$ \\
\hline Adverse Events & & $\mathrm{X}$ & $\mathrm{X}$ & & $\mathrm{x}$ & $\mathrm{X}$ & $\mathrm{X}$ \\
\hline
\end{tabular}


1. Identification of potential participants in hosting practices:

- Age 16-70yrs

- On asthma register

- Receipt of at least 1 prescription for asthma medication in the previous 12 months

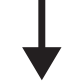

2. Mailed invitation to participate in study

- Study information and contact number

- Mini-AQLQ questionnaire to complete and return

3. Baseline assessment (at GP surgery), informed consent, randomisation

- Physiological measurements

- Questionnaire completion

- Randomisation to groups 1,2 and 3 (blinded to observer)

- Additional questionnaire completion (groups 1 and 3 only)

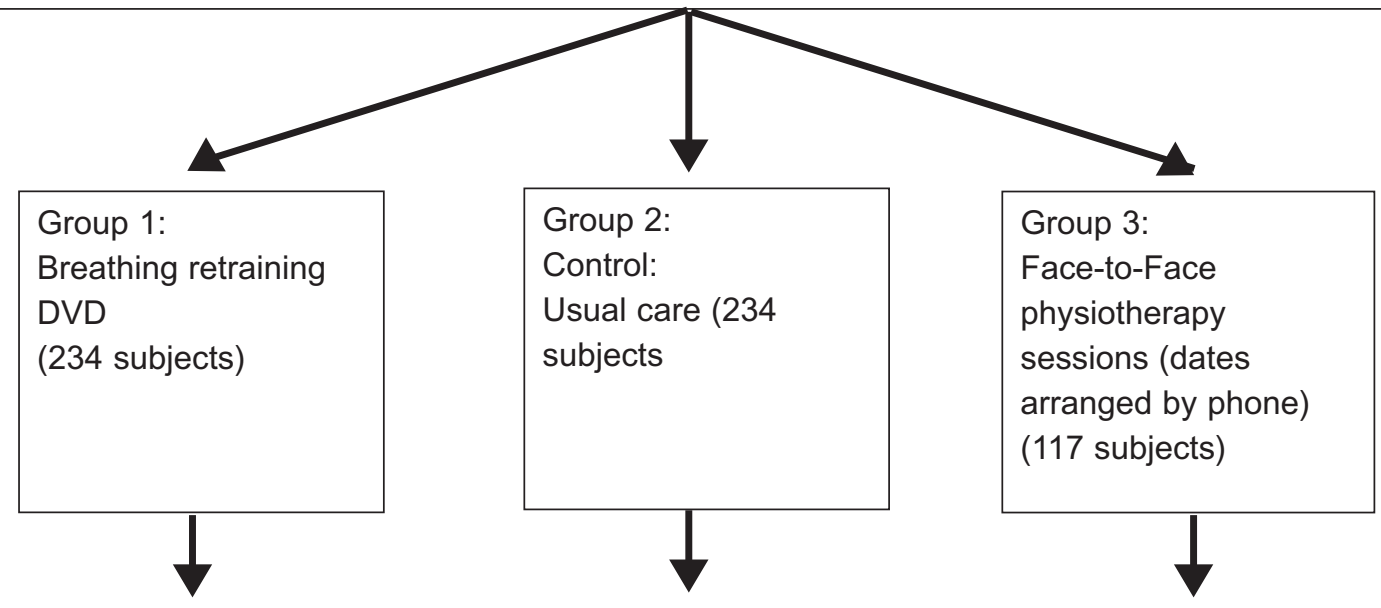

4. 3 months assessment:

- Questionnaires posted and completed
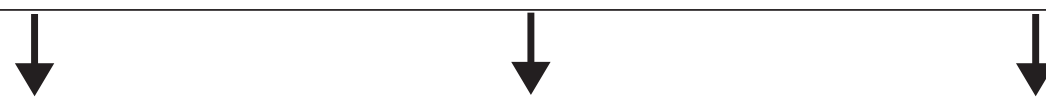

5. 6 months assessment:

- Questionnaires posted and completed
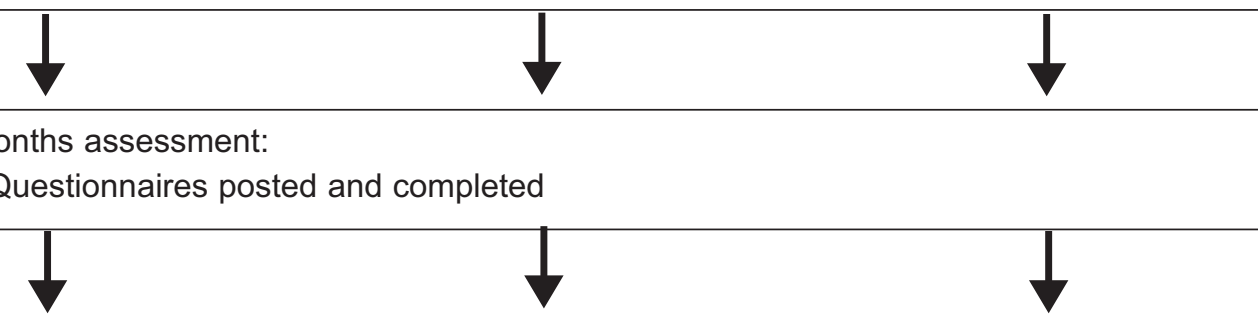

6. 12 months assessment (at GP surgery)

- Physiological measurements

- Questionnaire completion

- Health resource utilization over previous 12 months 